\title{
El Aprendizaje Afectivo y la Gamificación en Escenarios de Educación Virtual
}

\author{
Diego S. Melo-Solarte ${ }^{(1,2) *}$ y Paula A. Díaz ${ }^{(2,3)}$ \\ (1) Univ. de Manizales, Fac. Ciencias e Ingeniería, Cra 9 Nro 19-03, Manizales - Colombia \\ (2) Centro de Educación a Distancia (CEDUM), Cra 9 Nro 19-03, Manizales - Colombia \\ (3) Univ. de Manizales, Fac. Ciencias Sociales y Humanas, Cra 9 Nro 19-03, Manizales - Colombia \\ (e-mail: mdiego@umanizales.edu.co; pauladg@umanizales.edu.co)
}

* Autor a quien debe ser enviada la correspondencia

Recibido Sep. 27, 2017; Aceptado Nov. 27, 2017; Versión final Ene. 26, 2018, Publicado Jun. 2018

\begin{abstract}
Resumen
Se presenta la construcción de un entorno virtual de aprendizaje, que involucra en su diseño, elementos del aprendizaje afectivo y la gamificación de actividades, buscando crear un entorno amigable que responda a necesidades emotivas de los usuarios. Considerando que los entornos virtuales de aprendizaje deben dejar de ser considerados escenarios fríos y rígidos, dedicados exclusivamente a compartir contenidos y realizar actividades; es necesario que estos consideren las características que inciten o motiven al estudiante a realizar sus labores en pro de construir su conocimiento y desarrollar sus competencias. Además se describe la ruta metodológica para lograr la construcción del entorno virtual y posteriormente su validación a través de un estudio de caso donde se trabajó con estudiantes de educación media pertenecientes a escuelas rurales. Estos estudiantes, en su mayoría, no habían tenido experiencias que los vincule con procesos educativos a través de medios virtuales. Sin embargo, se logró que el $80 \%$ de los estudiantes lograra terminar el curso y dentro de ellos el 59\% mostró una curva ascendente en el aprendizaje.
\end{abstract}

Palabras clave: educación virtual; entorno virtual de aprendizaje; gamificación; aprendizaje afectivo; conciencia en las interfaces

\section{Emotional Learning and Gamification in Virtual Education Environments}

\begin{abstract}
This article presents the study and the process carried out for the construction of a virtual learning environment, which involves in its design elements of affective learning and gamification of activities trying to create a friendly environment that respond to the emotional needs of users. Considering that virtual learning environments should no longer be considered cold and rigid scenarios, dedicated exclusively to share content and activities, it is necessary that these consider the characteristics that induce or motivate the student to carry out their learning process and development of competences. This document describes the methodological route carried out in the project to achieve the construction of the environment and later its validation through a case study where we worked with students of basic education belonging to rural schools. Most of the students did not have experiences that link them to educational processes through virtual means. However, $80 \%$ of the students managed to finish the course and $59 \%$ of them showed an upward curve in learning.
\end{abstract}

Keywords: e-learning; virtual learning environment; gamification; affective learning; awareness 


\section{INTRODUCCIÓN}

Desde hace un par de décadas, la educación a distancia ha tenido un fuerte empujón con el auge de internet y con la gama de opciones que este medio permite, pues ello ha posibilitado la aparición de una variante conocida como "educación virtual" o "e-learning", que lo que busca es permitir el desarrollo de procesos educativos a través del ciberespacio (Bonilla, 2016), dejando de lado las limitantes de espacio y tiempo. Sin embargo, paulatinamente comienzan a surgir nuevas exigencias y nuevos retos, uno de ellos se enfoca en la creación de un escenario ideal, dispuesto para el desarrollo del proceso académico, el cual no solamente debe dar respuesta a unos requerimiento técnicos y procedimentales, sino que también debe considerar elementos funcionales o no funcionales, que contribuyan en la efectividad del proceso, es decir, el entorno virtual debe comenzar a tener en cuenta todos aquellos factores que pueden incidir en el desempeño de un estudiante y en la continuidad que él tenga para llevar a feliz término el proceso educativo, siempre en pro de cumplir con los objetivos de formación y procurando un rendimiento académico alto. Se puede decir que el éxito del proceso educativo a través de escenarios en línea, depende en gran medida de la aceptación que tengan los estudiantes sobre el entorno virtual y el modelo educativo (Blanco et al., 2016).

Varias investigaciones buscan definir los elementos clave con los cuales deben contar los entornos virtuales de aprendizaje con la intención de afianzar el proceso, entre ellos está la calidad de los contenidos, la flexibilidad, la posibilidad de personalización y de identificación del progreso como lo afirma (Esteban et al., 2016); pero también hay que tener en cuenta que estos ambientes además deben considerar factores humanos como los sicológicos o sociológicos que inciden directa o indirectamente en el proceso como lo sugiere (Hernández et al., 2016), pues es claro que hoy en día con la madurez que va adquiriendo la educación a través de medios electrónicos conlleva a pensar en elementos que pueden aportar en un proceso educativo que arroje buenos resultados para todos. En este proyecto se analizaron diferentes factores que pueden apoyar el proceso de formación en línea y que están asociados a la emotividad de los estudiantes, por ejemplo: motivación, curiosidad, necesidad, compromiso, entre otros, para ello se ha considerado las bases del aprendizaje afectivo (Valencia, 2014) y la gamificación de actividades educativas (Posada, 2013), como elementos que pueden aportar en el proceso de enseñanza y aprendizaje en entornos virtuales, considerando que el estudiante puede ser influenciado y motivado en cualquier momento del proceso y que las tecnologías pueden convertirse en un elemento que facilita la innovación de estrategias en estos campos.

Desde la perspectiva de creación del entorno de aprendizaje virtual, existen riesgos inherentes a la construcción de un nuevo software como lo plantea (Mascheroni y Greiner, 2012), muchos productos de software que se desarrollan no se culminan satisfactoriamente o si se hace, los usuarios no terminan utilizándolos porque no están cómodos o comprometidos con su aplicación, razón por la cual pensar en el diseño participativo, es una estrategia vinculante que puede afianzar la relación entre el aplicativo y los usuarios, por tal motivo, para la construcción del entorno virtual de aprendizaje se utilizaron las metodologías ágiles de diseño de software como en la creación de prototipos, además como mecanismo para identificar y analizar los elementos afectivos que puedan ser considerados dentro de un entorno virtual, se utilizó la Semiótica Organizacional (Liu, et al., 2012), estas metodologías planteadas por el método de articulación de problemas, permiten considerar las soluciones tecnológicas vistas desde las competencias y cualidades de los usuarios, estas estrategias se articularon con actividades de diseño participativo a través de grupos focales, donde los usuarios finales pudieron emitir sus ideas y opiniones frente a sus necesidades y a cómo ellos visualizan un entorno educativo virtual.

Como parte del proceso investigativo que se expone en este documento, se creó un entorno virtual de aprendizaje el cual considera la construcción de escenarios con elementos motivacionales para dinamizar los procesos de aprendizaje en línea, una vez dispuesto el entorno, se realiza un estudio de caso en el cual se involucran estudiantes de educación media pertenecientes al sector rural y con baja experiencia en tecnologías, especialmente en el uso de entornos virtuales para el aprendizaje; finalmente el documento presenta los resultados del caso y expone sus conclusiones.

\section{REFERENTES TEÓRICOS}

Con el fin de caracterizar los pilares teóricos que dan forma a esta investigación y a la vez que permiten concretar o unificar una definición que fue usada a lo largo del desarrollo del proyecto, se presentan los principales marcos conceptuales de este proyecto: entorno virtual para el aprendizaje, aprendizaje afectivo y gamificación.

\section{Entornos virtuales para el aprendizaje}

Con el auge de Internet, paulatinamente han ido surgiendo un sinnúmero de actividades para poder ser realizadas en el ciberespacio, y la educación no es ajena a ello, lo cual perfectamente nos lleva a hablar de 
la educación a distancia y cuando se habla de esta modalidad educativa se entiende que se incluye a las metodologías tradicional o virtual, cuya base de trabajo e interacción son los ambientes educativos que operan en Internet; por otra parte, están la educación presencial que bajo esquemas metodológicos usan escenarios en línea para el desarrollo de ciertas actividades, son estas las metodologías mixtas que también aprovechan las tecnologías como mecanismo de apoyo para comunicación e interacción.

Son varias las denominaciones y metodologías que se encuentran al hablar de educación mediada por las tecnologías: educación a distancia o educación virtual (e-learning), educación para apoyo a la presencialidad (b-learning), educación para móviles (m-learning) entre otras; sin embargo, todas estas metodologías tienen en común que requieren escenarios adecuados para apoyar su correcta operación, dichos escenarios son los denominados entornos virtuales para el aprendizaje (EVA), los cuales buscan ambientar un entorno de trabajo disponible en el ciberespacio, el cual debe estar adecuado con herramientas que apoyen el proceso metodológico en procura de lograr una educación efectiva.

Según (Mueller y Strohmeier, 2011), los EVA son bastante heterogéneos y cambian con el tiempo y el enfoque deseado, pueden ser entendidos como sistemas de información para apoyar las actividades administrativas y didácticas de los procesos de aprendizaje en la educación, (Salinas, 2012) resalta la importancia de estos entonces como mediadores del proceso de aprendizaje, además aduce que cada EVA deber operar de forma diferente según la metodología usada, es decir, como apoyo a los cursos presenciales o para aquellos que son totalmente virtuales; además señala la importancia de cambiar el pensamiento de docentes y estudiantes a la hora de interactuar con un sistema como estos, de aquí que (Valverde y Garrido, 2012), resalte la importancia de intervención del tutor como parte direccionadora y guía del proceso de aprendizaje. Los entornos de aprendizaje no deben ser espacios inertes, por el contrario deben permitir la creación de escenarios dinámicos, que promuevan el aprendizaje colaborativo (Guitert y Pérez-Mateo, 2013), es decir, donde todos los participantes puedan aportar desde su experiencia y habilidades, en la dinámica de construcción de nuevos conocimientos; además los EVA deben promover la autorregulación (Cabero, 2013) lo cual indica, pasar de usar estos entornos como simples herramientas tecnológicas a ser usadas como herramientas pedagógicas, a través de una acción planificada y organizada para alcanzar un objetivo determinado.

Si bien en el mercado existen Sistemas Gestores de Contenidos orientados al componente educativo (CMS), para tornarlos en EVA, se requiere de su adecuación para soportar una estructura metodológica que apoye el proceso de aprendizaje y una estructura operativa que apoye la gestión administrativa; por otra parte, se requiere de ciertas funciones como: la construcción y evaluación de documentos, el trabajo en grupo, guías para el proceso de aprendizaje, entre otras, que permiten apoyar el proceso y tornarlo efectivo, promoviendo el compromiso a lo largo del proceso de aprendizaje, la disciplina para cumplir con las labores, la conciencia de los avances y debilidades, posibilitando la interacción con otros y el trabajo colaborativo, de esta manera, y a pesar de que los EVA son sistemas computacionales, deben permitir, influenciar y considerar los factores humanos, los cuales de alguna forma inciden en el proceso formativo.

\section{Aprendizaje afectivo}

Las tecnologías están produciendo un gran cambio en la forma de estudiar, en la forma de interactuar en un curso, en la forma de impartir instrucciones, en la forma de aprender, en fin, en todos los aspectos asociados a la formación de una persona; todo parte de la forma como se llega al conocimiento, claro, hoy en día es diferente, pues los escenarios virtuales brindan nuevas y diferentes posibilidades, sin embargo, es claro que muchos de esos espacios adolecen de la intervención humana, dejando casi la totalidad del proceso a una retroalimentación automática, esquemática y fría, tornándose en gran parte, como factores causantes de la deserción y del bajo rendimiento académico (Estrada, 2014), si bien es cierto, los escenarios virtuales están llamados a innovar y motivar la acción educativa. Considerando al estudiante de manera integral, sea en escenarios presenciales o virtuales, es claro que el componente emocional juega un aspecto relevante a lo largo del proceso de formación colocando al mismo nivel tanto lo afectivo como lo cognitivo como lo afirma (Picard et al., 2004), de esta manera es correcto decir que las tecnologías juegan un importante papel, sin embargo están llamadas a ambientar escenarios motivadores y que promuevan la autorregulación.

No ha sido posible encontrar una definición concreta sobre el aprendizaje afectivo, sin embargo en la literatura se visualiza como un modelo emergente y contemporáneo, que por su carácter dinámico y complejo está en permanente construcción y resignificación, además se puede decir que se convierte en un modelo educativo adaptable a los diferentes contextos, humanos, sociales y culturales. El concepto planteado por (Ruiz, 2006), nos plantea que es imposible desconocer el papel de la emoción como moduladora y estabilizadora de los procesos de aprendizaje. El afecto es una auténtica dimensión de lo humano: Lo que nos caracteriza y diferencia de la inteligencia artificial es la capacidad de emocionarnos, de 
reconstruir el mundo y el conocimiento a partir de los lazos afectivos que nos impactan; de esta manera, el aprendizaje afectivo está asociado al componente emotivo del ser humano, siendo así, se puede decir que la capacidad de aprender que tienen las personas se ve influenciado directamente por sus emociones, lo cual va de la mano con lo planteado por (Valencia, 2014), el cual lo define como un proceso dinámico y dialógico que coloca al aprendiz de forma autónoma y contextuada, en una tensión permanente entre sus condiciones intelectuales y afectivas y las formas de responder coherente y consonante a las exigencias de su entorno.

\section{Gamificación}

"La Gamificación en un anglicismo, que proviene del inglés gamification, y que tiene que ver con la aplicación de conceptos que encontramos habitualmente en los videojuegos, u otro tipo de actividades lúdicas, como los videojuegos" (Cortizo et al., 2011). Todas las áreas o actividades de la vida cotidiana son susceptibles de aplicar los conceptos de gamificación, bajo este planteamiento, lo que busca este concepto dentro de los entornos educativos, es trasladar lo positivo de la mecánica de los juegos a los escenarios educativos, para el caso de los entornos virtuales de aprendizaje, se debe pensar en diseñar tareas y actividades con la finalidad de aprovechar la predisposición psicológica hacia el juego buscando inducir y mejorar la motivación hacia los aprendizajes (Posada, 2013), adicionalmente, la gamificación permite centrar la atención y el enfoque, por lo cual se traduce en un mecanismo propicio para mediar en procesos educativos (Arnold, 2014).

Para (Sánchez, 2015), utilizar el juego para el desarrollo de actividades serias, es un excelente mecanismo para incrementar la concentración, el esfuerzo y la motivación; si bien es cierto, la educación mediada por las tecnologías es una realidad, se requiere utilizar estrategias que contribuyan en el efectivo desarrollo del proceso, siendo así, la gamificación se convierte en una excelente opción para abocar a los estudiantes a llevar un hilo conductor y regulado dentro de su proceso. De acuerdo con (Moll, 2014), el concepto de gamificación se orienta al uso de las mecánicas de juego como competencias, premios, metas, niveles, entre otros, en entornos ajenos al juego, además resalta que considera disciplinas complementarias como es la sicología, que para el caso de la educación, trata a través del juego, de persuadir directa o indirectamente a los estudiantes para el cumplimiento de sus objetivos; por otra parte (Deterding et. Al, 2011) plantea en su investigación, el uso de elementos de diseño de juegos en contextos no relacionados con juegos, es decir, la gamificación no puede ser pensada solo con fines lúdicos sino también con fines productivos.

Los entornos virtuales de aprendizaje, comúnmente son descalificados por ser escenarios fríos y desmotivantes, sin embargo, algunas instituciones con experiencia en el área, como es el Observatorio de Innovación Educativa del Tecnológico de Monterrey, plantea que se puede innovar el área educativa de los cursos virtuales a través de la gamificación, pues a través de ella se puede influir en el comportamiento, incrementar la motivación y favorecer la participación de los estudiantes (Esteban et al., 2016). Si bien, muchos pedagogos son radicales con el tema del juego en el ámbito educativo, otros tantos, miran con interés la posibilidad de dinamizar el proceso de formación a través de estrategias educativas basadas en el juego y rescatan que en los entornos virtuales se requiere de la motivación y persuasión para orientar al estudiante y tratar de que él cumpla con sus actividades en pro de conseguir los objetivos o competencias de un determinado curso, en términos educativos no todo está dicho, con las tecnologías debemos tener la mente abierta a usar y crear nuevas posibilidades.

\section{METODOLOGÍA}

El proceso investigativo se desarrolló teniendo en cuenta diferentes conceptos referentes de gamificación y afectividad en procesos educativos, además se consideraron prácticas de diseño gráfico y comunicación basados en conceptos dados por la interacción humano-computador, de esta manera la metodología plateada en este proyecto se conformó por cinco fases:

Fase 1: se analizaron los referentes teóricos que buscan aportar en la construcción de un entorno virtual de aprendizaje amigable y efectivo, para ello se contempló el aprendizaje afectivo, la gamificación, además de la usabilidad y accesibilidad en sistemas web; elementos que dieron pie a lo que se ha denominado como "activadores motivacionales", los cuales pueden ser vistos como ideas gráficas, interactivas y comunicativas que tendrá el entorno virtual.

Fase 2: orientada la interpretación del problema y la identificación de los requerimientos funcionales y no funcionales de los ambientes virtuales de aprendizaje, para ello se usó el método de articulación de problemas (PAM)(Melo-solarte y Uribe, 2013) que hace parte de la Semiótica Organizacional, con el cual se busca caracterizar e interpretar un problema desde diferentes perspectivas, como es el caso de la 
identificación de las partes interesadas a través de las diferentes capas o niveles del modelo: El nivel operacional identificando sus componentes y funcionalidad; el nivel de contribuyentes considerando los responsables o actores del proceso; el nivel fuente, donde se contemplan los clientes y proveedores; el nivel mercado, donde se identifican los aliados, socios o la competencia y, finalmente, el nivel social, donde está el entorno conjuntamente con la legislación. Adicionalmente para el análisis de requerimientos del ambiente virtual de aprendizaje visto desde la perspectiva del estudiante, fueron usados otros dos artefactos más, el cuadro de evaluación con el cual se plantean problemáticas de menor orden y se plantean alternativas de solución; finalmente el marco de referencia semiótico con lo cual se hace una evaluación de las plataformas tecnológicas y su relación con los factores humanos.

Fase 3: identificados los requerimientos afectivos y de gamificación, se procede a la creación de las maquetas que contemplan los elementos gráficos y comunicativos del entorno virtual, con ello se evalúa la viabilidad de cada concepto y se procede a la creación de prototipos no funcionales a fin de materializar las ideas planteadas en la primera fase.

Fase 4: una vez determinada la viabilidad de cada elemento, se procedió a la creación de un prototipo funcional con lo cual se aspira a tener una visión más clara de su funcionalidad, a partir de esta instancia se posibilita el análisis de usabilidad y accesibilidad considerando al usuario final y utilizando las metodologías de evaluación heurística y de rastreo ocular donde se determina el punto exacto donde se enfoca el usuario. Cabe resaltar que la usabilidad de una aplicación web y/o en una plataforma educativa, "es un criterio importante en el rendimiento y calidad del producto software, y aunque su evaluación puede tener un componente subjetivo, el estudio de normas, guías, estándares y diferentes propuestas permite unificar los aspectos relevantes" (Alarcón-aldana, et al. 2014) asociados a la necesidad del aplicativo, pero que también garantiza que los usuarios se sientan cómodos con su interacción.

Fase 5: Durante el proceso investigativo, se busca validar los diferentes elementos que han sido incorporados al ambiente virtual como elementos orientados a dinamizar y motivar en el proceso de aprendizaje, para ello se ha implementado un caso de estudio con ocho instituciones educativas de educación media, pertenecientes al sector rural de Colombia.

\section{CONSTRUCCIÓN DE UN ENTORNO VIRTUAL MOTIVADOR}

Se presentan los principales elementos considerados en la implementación de un entorno virtual que motive el proceso académico, a la vez se indica el procedimiento llevado a cabo para su diseño e implementación.

\section{Consideraciones afectivas y gamificadas}

Dentro del proceso adelantado en esta investigación fue posible identificar diferentes elementos que pueden ser implementados dentro de un entorno virtual de aprendizaje, algunos de ellos en el área de aprendizaje afectivo, otros en el área de la gamificación, sin embargo también se pudieron identificar elementos que debían ser abordados desde la concepción de "awareness", es decir considerando la posibilidad de que el usuario conozca y tenga conciencia de la interfaz o su entorno y su posición en él, tratando de lograr en el usuario, una postura consiente de su estado a partir de la comparación con los demás, elementos que también pueden llegar a incidir en la motivación de su participación. Cabe resaltar que el foco de la pesquisa está orientado en la construcción de entornos virtuales, que contribuyan en la dinámica de un curso y a la vez generen en los estudiantes diferentes reacciones de índole motivacional, en aras de persuadir y generar autorregulación en el aprendizaje, a continuación, se presenta los principales elementos que fueron incorporados en este entorno al cual se le denominó “Kava Estudiante" ver Tabla 1.

Los elementos motivaciones incorporados en la Tabla 1, se clasifican en tres tipos: Gamificación, si es un componente orientado al aprendizaje mediado por el juego o por la lúdica; aprendizaje afectivo, teniendo en cuenta que sea un elemento orientado a motivar o persuadir al estudiante; finalmente, el tipo denominado conciencia del entorno, el cual determina si es un componente incorporado para que el estudiante conozca su estado y el de sus compañeros.

\section{Diseño e implementación de interfaces}

Dentro de la construcción de un entorno virtual para educación, existen varios aspectos de tipo pedagógico y tecnológico que deben ser considerados para el correcto desarrollo de un curso, sin embargo, en este proyecto se buscó dilucidar elementos asociados al aprendizaje afectivo, gamificación e incluso Conciencia de entorno, que contribuyan desde la persuasión y motivación, para el correcto desarrollo de un curso virtual por parte de los estudiantes. Adicionalmente, en el diseño de las interfaces se consideraron aspectos 
propios del desarrollo de software, con lo cual se incorporan conceptos como: Diseño basado en grilla para determinar la distribución de los elementos en pantalla y la forma como esos elementos pueden agruparse; por otra parte se considera aspectos como el color, usando en los diseños colores fríos para minimizar el cansancio en las lecturas; finalmente se considera el uso de patrones de desarrollo de software, tal es el caso del patrón Z, que recalca sobre la tendencia que tienen las personas para leer de izquierda a derecha y de arriba a abajo, influyendo en la forma como se percibe la pantalla (Acevedo, 2010).

Tabla 1: Elementos motivacionales incorporados en Kava estudiante

\begin{tabular}{|c|c|c|c|}
\hline Activador & Descripción & Tipo & Aplicación \\
\hline $\begin{array}{l}\text { Quien quiere ser } \\
\text { millonario }\end{array}$ & $\begin{array}{l}\text { Modificar la presentación de los cuestionarios involucrando } \\
\text { elementos de gamificación a través del formato quien quiere } \\
\text { ser millonario. }\end{array}$ & Gamificación & Actividades \\
\hline Agente virtual & $\begin{array}{l}\text { Un avatar con la posibilidad de tener movimientos emotivos y } \\
\text { mostrar mensajes de texto y audio informativos o emotivos, } \\
\text { con la intencionalidad de generar la sensación de } \\
\text { acompañamiento y emoción cuando el avatar aparezca. }\end{array}$ & $\begin{array}{l}\text { Aprendizaje } \\
\text { afectivo }\end{array}$ & $\begin{array}{l}\text { Entorno } \\
\text { virtual }\end{array}$ \\
\hline $\begin{array}{l}\text { Cumplimiento de } \\
\text { actividades }\end{array}$ & $\begin{array}{l}\text { Mecanismo que permita visualizar el cumplimiento a la hora } \\
\text { de realizar una actividad, posibilitando que todos los alumnos } \\
\text { puedan conocer el estado general y personal. }\end{array}$ & $\begin{array}{l}\text { Conciencia } \\
\text { de entorno }\end{array}$ & Actividades \\
\hline Progreso del curso & $\begin{array}{l}\text { La interfaz general que indique todos los componentes del } \\
\text { curso y cómo va la evolución de cada componente (usar } \\
\text { mapa de colores verde, naranja, gris, rojo). }\end{array}$ & $\begin{array}{l}\text { Aprendizaje } \\
\text { afectivo }\end{array}$ & $\begin{array}{l}\text { Entorno } \\
\text { virtual }\end{array}$ \\
\hline $\begin{array}{l}\text { Visualizador de } \\
\text { contenidos textuales }\end{array}$ & $\begin{array}{l}\text { Mecanismos para visualizar los contenidos usando el efecto } \\
\text { de pasando páginas en un libro. }\end{array}$ & Gamificación & Contenidos \\
\hline Mapa de etiquetas & $\begin{array}{l}\text { Mecanismo para generar un mapa de etiquetas a partir de } \\
\text { cada lectura o contenido textual. }\end{array}$ & Gamificación & Contenidos \\
\hline $\begin{array}{l}\text { Mapa de } \\
\text { colaboración en foros }\end{array}$ & $\begin{array}{l}\text { Generador de mapa de colaboración, es decir un diagrama } \\
\text { interactivo donde se pueda reflejar las relaciones de } \\
\text { colaboración y participación entre estudiantes. }\end{array}$ & $\begin{array}{l}\text { Conciencia } \\
\text { de entorno }\end{array}$ & Foros \\
\hline Audios & $\begin{array}{l}\text { Todos los textos creados se presentarán en formato de audio } \\
\text { narrados por profesionales y de forma emotiva. }\end{array}$ & $\begin{array}{l}\text { Aprendizaje } \\
\text { afectivo }\end{array}$ & Contenidos \\
\hline $\begin{array}{l}\text { Recordatorios vía } \\
\text { sms }\end{array}$ & $\begin{array}{l}\text { Mecanismo para enviar mensajes emotivos y recordatorios de } \\
\text { las actividades que el estudiante tiene pendientes. }\end{array}$ & $\begin{array}{l}\text { Aprendizaje } \\
\text { afectivo }\end{array}$ & Actividades \\
\hline $\begin{array}{l}\text { Actividades } \\
\text { valorativas basadas } \\
\text { en juegos }\end{array}$ & $\begin{array}{l}\text { Implementar cuestionarios usando lógicas de juegos como: el } \\
\text { ahorcado, sopa de letras y concéntrese, esto aplica para las } \\
\text { actividades de ejercitación del estudiante. }\end{array}$ & Gamificación & Actividades \\
\hline Control de lectura & $\begin{array}{l}\text { Se harán preguntas cada cierto contenido, esto permitirá } \\
\text { ganar puntos y adicionalmente activar el resto del texto. }\end{array}$ & Gamificación & Contenidos \\
\hline Ganancia de escudos & $\begin{array}{l}\text { Se crea la metáfora de ganancia de escudos a partir del } \\
\text { control de lectura, cuando un estudiante tenga los escudos } \\
\text { necesarios, podrá activar un video o un juego como premio. }\end{array}$ & Gamificación & $\begin{array}{l}\text { Entorno } \\
\text { virtual }\end{array}$ \\
\hline Ranking de usuarios & $\begin{array}{l}\text { En la interfaz principal de cada módulo se dispondrá de un } \\
\text { sistema de posicionamiento que permite ver cuál es el estado } \\
\text { de avance para todos los participantes. }\end{array}$ & $\begin{array}{l}\text { Conciencia } \\
\text { de entorno }\end{array}$ & $\begin{array}{l}\text { Entorno } \\
\text { virtual }\end{array}$ \\
\hline Mi posición & $\begin{array}{l}\text { Basado en las actividades desarrolladas, permite mostrar el } \\
\text { estado de avance del módulo para el estudiante, } \\
\text { adicionalmente se muestra los escudos que ha logrado }\end{array}$ & $\begin{array}{l}\text { Conciencia } \\
\text { de entorno }\end{array}$ & $\begin{array}{l}\text { Entorno } \\
\text { virtual }\end{array}$ \\
\hline Control de entrega & $\begin{array}{l}\text { Mecanismo para identificar cómo va el cumplimiento de las } \\
\text { actividades a nivel individual y a nivel grupal, reflejando el } \\
\text { porcentaje de estudiantes que han cumplido y el porcentaje } \\
\text { de estudiantes pendientes. }\end{array}$ & $\begin{array}{l}\text { Conciencia } \\
\text { de entorno }\end{array}$ & Actividades \\
\hline
\end{tabular}




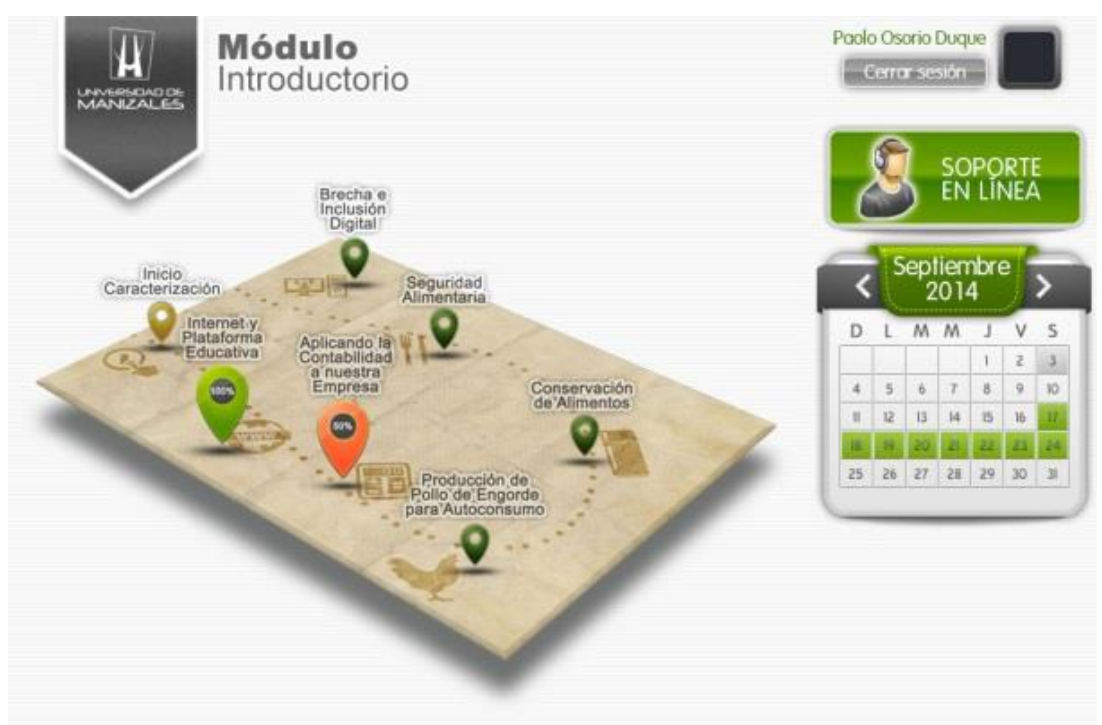

Fig. 1: Interfaz nivel 0 - panorámica general del curso

De esta manera y considerando los elementos identificados a lo largo del proceso investigativo, se construyó una entorno virtual que opera en tres niveles, el primero, es un nivel donde se muestran el panorama de todos los módulos disponibles en el curso y la secuencia o ruta que se debe seguir (ver Fig. 1); en esta interfaz se puede evidenciar la evolución porcentual del estudiante en cada módulo y se usa un esquema de colores para evidenciar el estado en el que se encuentra un módulo. Una vez el estudiante ingresa a cada módulo, encuentra el segundo nivel (como lo muestra la Fig. 2); en esta interfaz se presenta toda la estructura metodológica e interactiva del módulo, en la parte central están cinco componentes orientados al componente disciplinar y al desarrollo de los contenidos temáticos (prueba de entrada, generalidades, criterios conceptuales, actividades de aprendizaje y pruebas de salida), en la parte derecha están cuatro bloques orientados a la parte interactiva (navegación, foros, recursos y administración), finalmente, en la parte inferior del módulo se está implementado un ranking que permite identificar el avance del estudiante en ese módulo y cuál es su posición frente a otros compañeros en el mismo curso. Finalmente, el tercer nivel está orientado a la visualización de los diferentes contenidos multimedia que hacen parte del módulo y también posibilita el desarrollo de las diferentes actividades de aprendizaje.

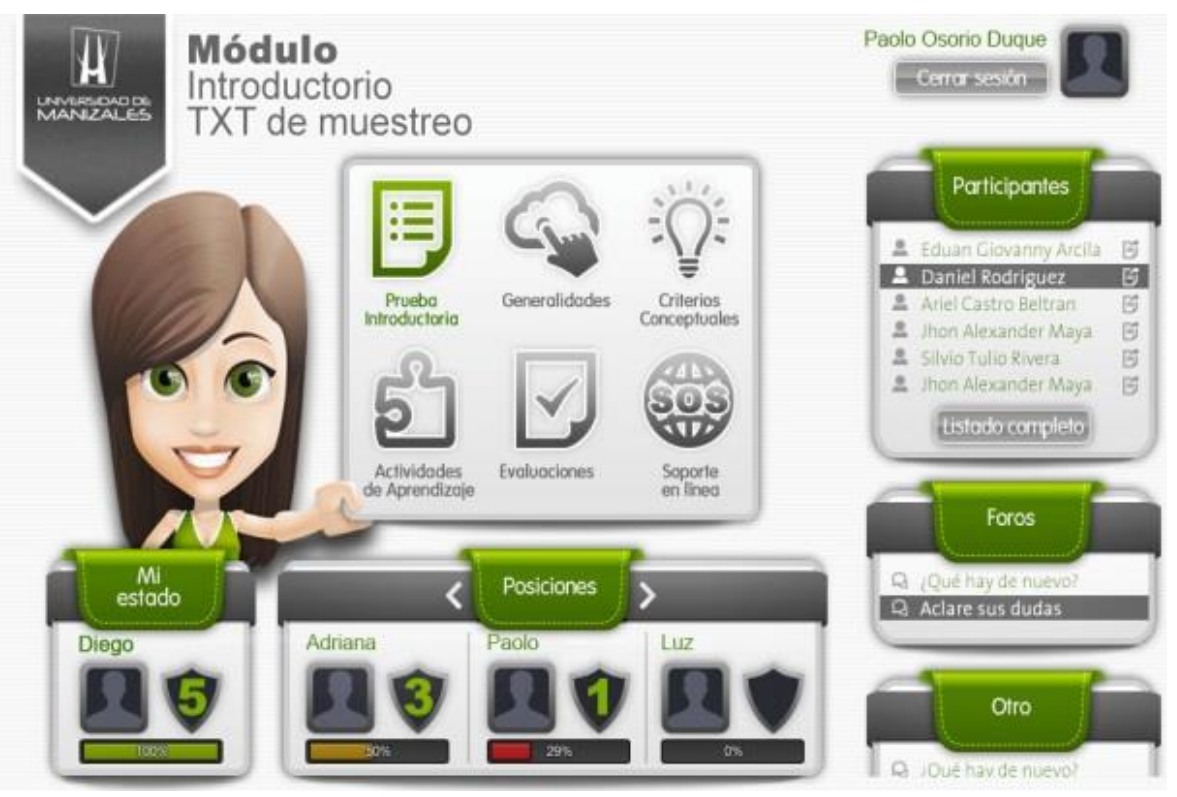

Fig. 2: Interfaz nivel 1 - estructura de un módulo

En cada nivel, según la necesidad, se implementó la funcionalidad de los diferentes activadores motivacionales, como elementos adicionales a la dinámica metodológica de un módulo y con lo cual se espera persuadir positivamente a los estudiantes, en pro de motivar su interacción con el entorno y a la vez que construye paulatinamente sus aprendizajes y competencias. 
Por otra parte, es necesario aclarar que en el proceso investigativo y en el trabajo de campo realizado, también se pudieron identificar necesidades sutiles dentro del diseño gráfico de una interfaz, tal es el caso de la prevalencia de un avatar de carácter femenino para mostrar la estructura del módulo y también un agente virtual para emitir los mensajes de información o motivación; el carácter femenino del avatar se obtuvo a partir de una encuesta realizada con los estudiantes, donde el $92 \%$ planteo que fuera femenino, según los estudiantes sienten mayor confianza con alguien que represente el género femenino; así mismo las tonalidades usadas también se fueron modificando a lo largo del proceso y en ello también tuvieron incidencia los estudiantes quienes manifestaban su aprobación o desaprobación por determinadas interfaces. Los entornos virtuales por lo general son considerados fríos y poco amigables, en gran medida enfocados a posibilitar la publicación de contenidos y el desarrollo de actividades de aprendizaje, sin embargo en esta propuesta, se consideraron elementos que buscan tornar un ambiente más afectivo y que en el fondo ayude en la autorregulación del estudiante, generando la sensación de acompañamiento y así evitando su deserción académica.

\section{ESTUDIO DE CASO}

Como mecanismo de validación del entorno virtual y de los activadores motivacionales que allí se implementaron, se desarrolló un estudio de caso para lo cual se implementó en la plataforma un curso con seis módulos académicos, con temáticas alusivas al sector agropecuario, el test del entorno virtual incluyó el trabajo con seis instituciones educativas del sector rural ubicadas en los departamentos de Caldas, Cauca, Huila y Nariño, En esas instituciones se trabajó con ciento doce (112) estudiantes de los grados noveno, decimo y onceavo; a quienes se les concedió acceso para ingresar al entorno virtual y cuya meta era el desarrollo de dos módulos según su interés, con la intensión de validar la interacción en la plataforma propuesta.

Este proceso se realizó en seis pasos: 1) la consecución de las instituciones educativas del sector rural que tuvieran la intencionalidad de vincularse al proyecto; 2) el desarrollo de una reunión con docentes que ejercerían la labor de coordinadores del proceso que se llevaría a cabo en la institución educativa, esto con el fin de contextualizar el proyecto y determinar las funciones de cada coordinador; 3) el desarrolló una reunión con estudiantes familiarizando el proyecto y el ambiente virtual para dar inicio al proceso, además, en esta sesión se hizo una caracterización de la situación digital y un pre-análisis de interfaces usando un rastreador ocular, como se puede observar en la

Fig. 3 ; 4) el seguimiento y control al desarrollo del curso por parte de los estudiantes, quienes realizaron el módulo 'Internet y plataforma educativa' de forma obligatoria para familiarizarse con la metodología, posteriormente debieron escoger uno de cuatro módulos técnicos, según su intencionalidad, para el proceso completo cada estudiante tardó un mes, según lo planeado; 5) el desarrollo de una encuesta para identificar patrones de situación digital y conceptos sobre el uso de la plataforma educativa; 6) el análisis de resultados con el comportamiento de los estudiantes en el entorno virtual y con los resultados de la encuesta.

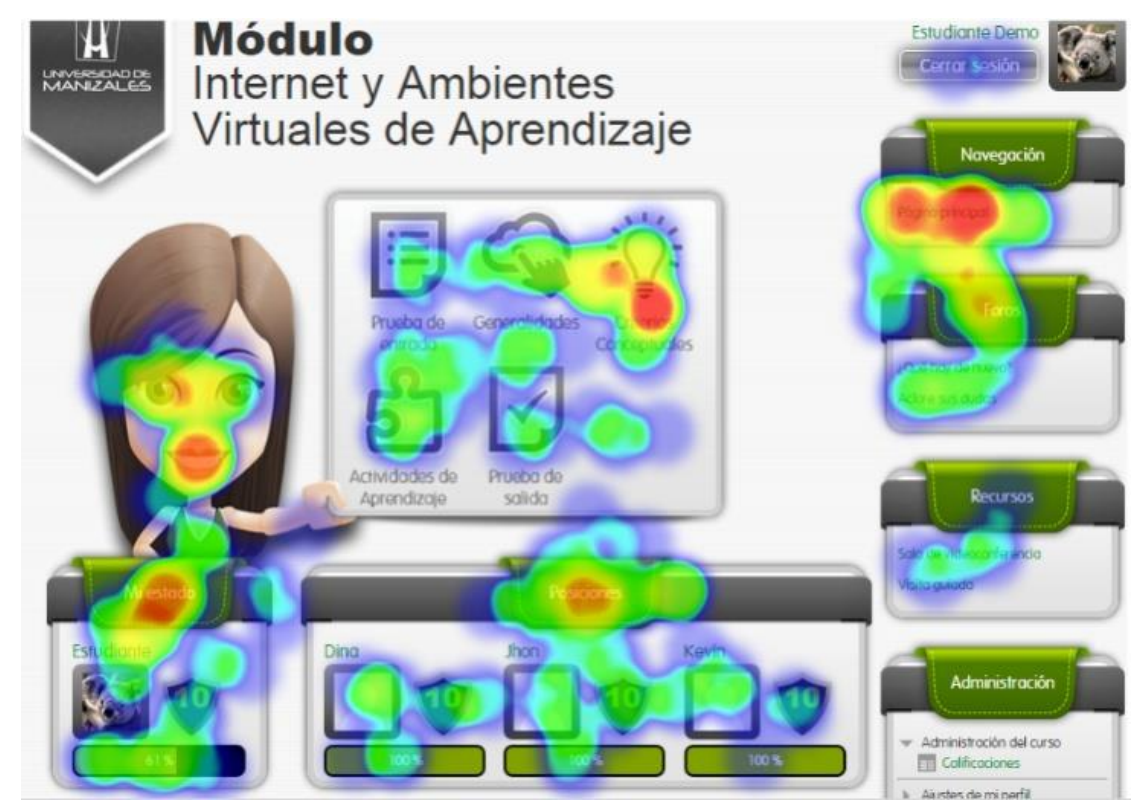

Fig. 3: Interfaz del módulo - mapa de calor 
Desde el punto de vista del desempeño y desarrollo de cada curso, se logró observar que el $80 \%$ de los estudiantes completaron los dos módulos propuestos dentro del tiempo pactado, mostrando que un $59 \%$ de ellos tuvo un aumento en el dominio de las temáticas trabajadas en cada módulo, logrando mejorar sus pruebas entre un $10 \%$ y un $25 \%$; el $17 \%$ de los estudiante se mantuvo en el mismo rendimiento, es decir no se evidenció progreso en las temáticas tratadas en los módulos, y el $22 \%$ presentó una reducción en sus resultados iniciales, esto contrastando la prueba inicial con la prueba final de cada módulo, advirtiendo que las dos pruebas tienen el mismo cuestionario.

Desde el punto visual e interactivo, la apreciación de los estudiantes es que es una plataforma sencilla e intuitiva, que es fácil de aprender la lógica metodológica, aunque si se hace necesaria la inducción inicial para poder entender cómo se desarrollará el proceso. Por esto se plantea la necesidad de tener una prueba de entrada y una prueba de salida, aunque la prueba de entrada atemoriza un poco, tal vez por prejuicios culturales y miedo a sentirse juzgado según lo manifiestan los mismos estudiantes; las dos pruebas son simples cuestionarios que buscan identificar en los estudiantes, los conocimientos previos o posteriores sobre la temática que se trabajará en cada módulo. Los estudiantes han expresado que al principio se perdieron de los contenidos presentados, pero conforme se fueron familiarizando, fue más fácil poderlos ubicar; indican también que hubo confusión con los íconos que presentaban contenidos multimedia adicionales, sugiriendo que mejor deberían estar inmersos como enlaces dentro de los textos o contenidos.

Desde la estimulación, pueden plantearse dos puntos de vista, uno, los efectos producidos por cada activador motivacional implementado dentro de la plataforma y, dos, del ambiente en general, es decir, la forma como se presentan los contenidos, la metodología utilizada, el acompañamiento, entre otros; lo cual refleja claramente que es un ambiente familiar y amigable, que perfectamente contribuye en la motivación del proceso de aprendizaje en un entorno virtual, aunque es claro que los estudiantes quieren un ambiente dinámico, que esté cambiando constantemente, pues al hacer un módulo con una estructura, ellos memorizan dicho mecanismo, pero al hacer el segundo módulo la curiosidad se comienza a perder.

Como se puede ver en la Tabla 2 , varios activadores motivacionales cumplieron su misión, que fue la de motivar y persuadir a los estudiantes en el desarrollo de un determinado curso, hasta el punto de provocar que el $60 \%$ tratara de cumplir con el curso en menos tiempo del propuesto, el $25 \%$ en el tiempo pactado, el $15 \%$ no lo terminó. Adicionalmente se detectó que un grupo reducido de 15 estudiantes ingresaron a la plataforma, posterior a la finalización del estudio de caso, mostrando su interés por las otras temáticas disponibles en la plataforma y porque manifiestan que es un ambiente agradable para estudiar.

Tabla 2: Comentarios sobre los activadores motivacionales

\begin{tabular}{|c|c|}
\hline Activador & Comentarios \\
\hline $\begin{array}{l}\text { Quien quiere ser } \\
\text { millonario }\end{array}$ & $\begin{array}{l}\text { Los estudiantes aprueban que los cuestionarios sean presentados de una manera lúdica y que } \\
\text { se implemente estrategias de gamificación. }\end{array}$ \\
\hline Agente virtual & $\begin{array}{l}\text { La implementación de un agente virtual con apariciones aleatorias, llamó mucho la atención, } \\
\text { se trató de reflejar un elemento animado gracioso y según los estudiantes despertó mucho la } \\
\text { curiosidad para identificar en que momentos aparecía. }\end{array}$ \\
\hline $\begin{array}{l}\text { Cumplimiento de } \\
\text { actividades }\end{array}$ & $\begin{array}{l}\text { Manifiesta que no es muy claro el mensaje que se transmite con esta herramienta, sin } \\
\text { embargo, una vez explicado manifiestan que puede ser de mucha ayuda. }\end{array}$ \\
\hline Progreso del curso & $\begin{array}{l}\text { Según los participantes, tener presente el porcentaje de cómo va su curso, genera un reto y } \\
\text { una curiosidad por identificar y realizar las actividades que les permitan aumentar dicho } \\
\text { porcentaje, algunos estudiantes manifiestan que los emociona: "el hecho es ver que cambie o } \\
\text { que suba". }\end{array}$ \\
\hline $\begin{array}{l}\text { Visualizador de textos } \\
\text { o documentos }\end{array}$ & $\begin{array}{l}\text { La presentación de los contenidos y la interacción se desarrolló en forma de libro lo cual fue } \\
\text { un elemento amigable y agradable para los estudiantes. }\end{array}$ \\
\hline Mapa de etiquetas & $\begin{array}{l}\text { Los mapas de etiquetas presentes en los documentos de texto no tuvieron trascendencia } \\
\text { dentro de la presentación de contenidos. }\end{array}$ \\
\hline Avatar & $\begin{array}{l}\text { Disponer de un 'avatar' tornó amigable la estructura de los módulos. Es claro anotar que hay } \\
\text { una fuerte inclinación a que el 'avatar' sea de sexo femenino, ya que demuestra mayor } \\
\text { carisma. }\end{array}$ \\
\hline $\begin{array}{l}\text { Audios con voz } \\
\text { masculina y femenina }\end{array}$ & $\begin{array}{l}\text { Los audios permitieron que los estudiantes, aparte de leer los textos, también se motivaran a } \\
\text { escucharlos convirtiéndose esto en un ejercicio de refuerzo de la comprensión lectora. }\end{array}$ \\
\hline
\end{tabular}


Tabla 2: Continuación.

\begin{tabular}{|c|c|}
\hline Reloj de arena & $\begin{array}{l}\text { El efecto del reloj no tuvo mayor relevancia en la plataforma, por lo cual, su funcionalidad fue } \\
\text { cambiada por mensajes presentados por el agente virtual animado. }\end{array}$ \\
\hline $\begin{array}{l}\text { Mensajes de audio } \\
\text { motivadores }\end{array}$ & $\begin{array}{l}\text { Los mensajes no se presentaron en audio, sino de una forma animada a través del agente } \\
\text { virtual, lo cual produjo buenos resultados dentro de lo que se podría entender en llamar la } \\
\text { atención al estudiante, para centrarlo en una actividad. }\end{array}$ \\
\hline $\begin{array}{l}\text { Incluir actividades de } \\
\text { aprendizaje lúdicas }\end{array}$ & $\begin{array}{l}\text { Los estudiantes prefieren que los cuestionarios evaluativos sean presentados de forma lúdica, } \\
\text { es decir, a manera de juego como el ahorcado, sopa de letras, concéntrese, etc. }\end{array}$ \\
\hline Control de lectura & $\begin{array}{l}\text { A los estudiantes les llamó la atención que los contenidos se visualizaran en su totalidad si se } \\
\text { respondía bien una pregunta sobre el texto inmediatamente anterior, este elemento motivó a } \\
\text { los estudiantes para concentrarse y poder responder la pregunta al primer intento. }\end{array}$ \\
\hline Ganancia de escudos & $\begin{array}{l}\text { Cuando un estudiante respondía las preguntas de control de lectura al primer intento, podía } \\
\text { ganar unos escudos que luego podrían ser cambiados por vidas de un juego, lastimosamente } \\
\text { se logró una implementación parcial de esta funcionalidad, provocando frustración en algunos } \\
\text { estudiantes. }\end{array}$ \\
\hline Ranking de usuarios & $\begin{array}{l}\text { Conocer el estado y los avances de todos los usuarios de un curso, provocó que todos } \\
\text { quisieran cumplir sus metas antes de lo previsto para llegar a los primeros lugares o estar al } \\
\text { menos en el promedio, sin embargo, se recomienda que para clasificar dicho ranking, se tenga } \\
\text { en cuenta a los estudiantes de una misma escuela sin combinarlos con otras instituciones. }\end{array}$ \\
\hline Mi posición & $\begin{array}{l}\text { Visualizar los avances del estudiante y tener la forma de confrontar con el ranking permitió que } \\
\text { el estudiante tuviera conocimiento de cuan avanzado o retrasado va en el proceso. }\end{array}$ \\
\hline Control de entrega & $\begin{array}{l}\text { Está metáfora ayudó al estudiante a tener claridad sobre el estado de su entrega y conocer } \\
\text { cómo estaban sus compañeros respecto al mismo tema. }\end{array}$ \\
\hline
\end{tabular}

Finalmente se encontró un dato importante frente al uso real de la plataforma, y es el tiempo efectivo que un estudiante permanece o requiere para desarrollar un módulo, encontrando que los estudiantes que lograron un buen desempeño en su prueba de salida, permanecieron entre 7 y 8 horas aproximadamente de tiempo efectivo, cursando dos módulos: un introductorio y uno con temática específica. Mientras los que estuvieron por debajo de esta media o por encima de ella, no reflejan una buena prueba de salida.

\section{CONCLUSIONES}

El ambiente virtual creado en este proyecto cumplió con el objetivo trazado, es claro que el proceso de enseñanza y aprendizaje en escenarios virtuales no es fácil para los estudiantes, además, en el rendimiento de un curso inciden muchas variables que motivan o desmotivan a los estudiantes, llevando en muchos casos al abandono del proceso, sin embargo, se pudo notar que la suma de cuatro elementos importantes, contribuyen en la permanencia y dedicación del proceso, son: 1) entorno virtual dinámico, simple y agradable; 2) espacios para interactuar con los compañeros académica y lúdicamente; 3) acompañamiento real o virtual; 4) actividades de aprendizaje novedosas y creativas. Estos requerimientos se pueden entender como la motivación que tienen las personas al desarrollar un proceso de enseñanza y aprendizaje, a esto se le puede sumar otros factores importantes para el éxito del mismo, son: la convicción y compromiso que deben tener las personas para emprender un curso en línea.

El diseño participativo y la Semiótica Organizacional se constituyen en estrategias ideales para la identificación de las necesidades del entorno educativo virtual, porque permite involucrar a los futuros usuarios, en el diseño de una propuesta interactiva y comunicativa del entorno, además, permite identificar de primera mano las necesidades que ellos tienen y cómo ellos mismos crean alternativas de solución, que por cierto, es claro que al verse inmersos en la creación de alternativas que solucionan el problema, permite lograr mayor grado de empoderamiento de dicha solución.

Dentro del desarrollo de este proyecto, se pudo percibir que involucrar elementos gamificados dentro de las interfaces, contribuyó favorablemente en la motivación de los estudiantes, para ellos descubrir cada vez algo nuevo en la interfaz induce constantemente a tratar de hacer algo para aprender y a su vez para tratar de que la plataforma les de algo a cambio; por otra parte, los elementos afectivos reflejados principalmente en aspectos comunicativos de las interfaces, ayudo a que los estudiantes se sientan en un ambiente agradable para trabajar y siempre se mostraban expectantes al posible mensaje que les muestre el avatar, ya sea como instrucción o como reconocimiento a su avance. 
A través del caso de estudio, se pudo evidenciar que el diseño y la construcción de un entorno virtual de aprendizaje, deben ir de la mano con el ser y el sentir de la persona, buscando generar escenarios que sean motivadores, que promuevan la sensación de acompañamiento y que induzcan al estudiante a comprometerse con el proceso y su autorregulación; se pudo notar que un estudiante que participa en la construcción del ambiente, se siente identificado y a la vez comprometido con las labores que se pueden desarrollar a través del entorno educativo

\section{AGRADECIMIENTOS}

Los autores agradecen el apoyo de la Universidad de Manizales a través del programa de investigación denominado 'Construcción de ambientes innovadores e inclusivos para el aprendizaje en escenarios virtuales', ejecutado mediante la alianza Universidad de Manizales - CHEC EPM, dentro de la convocatoria Colciencias-MEN 578-2012.

\section{REFERENCIAS}

Acevedo, C.P., J.L. Arciniegas, X. García y J. Perrinet; Proceso de Adaptación de una Aplicación de e-aprendizaje a taprendizaje. Información tecnológica, 21(6), 27-36. (2010).

Alarcón-aldana, A.C., E.L. Díaz y M. Callejas-cuervo; Guía para la evaluación de la Usabilidad en los Entornos Virtuales de Aprendizaje (EVA). Información tecnológica, 25(3), 135-144. (2014)

Arnold, B. J. (2014). Gamification in education. American Society of Business and Behavioral Sciences, 21(1) 32-39. Retrieved from https://search.proquest.com/docview/1519057772?accountid=42082, acceso: diciembre 2017, (2014)

Blanco, A., P.A. Fernández, y P. Clave; La perspectiva de estudiantes en línea sobre los entornos virtuales de aprendizaje en la educación superior, DOI: http://dx.doi.org/10.20548/innoeduca.2016.v2i2.1062,. INNOEDUCA. International journal of technology and educational innovation, 2(2), 109-116 (2016)

Bonilla, L.A.; Deliberación entorno a la Educación Virtual, http://revistas.uv.mx/index.php/IS/article/view/1112, Interconectando Saberes, 1(1), 77-89 (2016)

Cabero, J.; El aprendizaje autorregulado como marco teórico para la aplicación educativa de las comunidades virtuales y los entornos personales de aprendizaje,

http://campus.usal.es/ revistas_trabajo/index.php/revistatesi/article/view/10217/10626, ISSN: 2444-8729, Teoría de la

Educación. Educación y Cultura en la Sociedad de la Información (TESI), 14(2), 133-156 (2013)

Cortizo, J.C., F. Carrero, B. Monsalve, A. Velasco, L.I Díaz y J. Pérez; Gamificación y Docencia : Lo que la universidad tiene que aprender de los videojuegos, VIII Jornadas Internacionales de Innovación Universitaria, 1-8, Madrid, España, 11 y 12 de julio (2011)

Deterding S., D. Dixon, R. Khaled y L. Nacke, From game design elements to gamefulness: defining "gamification", 15th International Academic MindTrek Conference: Envisioning Future Media Environments, (2011)

Esteban, M., A.B. Bernardo, L. Rodríguez, R. Cerezo, J.C. Nuñez y A. Casaravilla; Claves para facilitar el éxito en Entornos Virtuales de Aprendizaje, VI Conferencia Latinoaméricana Sobre el Abandono en la Educación Superior (CLABES), 1-7, Quito, Ecuador 9 al 11 de noviembre (2016).

Estrada, R.; Blended-Learning afectivo y las herramientas interactivas de la web 3.0: Una revisión sistemática de la literatura, http://eticanet.org/revista/index.php/eticanet/article/view/45/39, ISSN: 1695-324X, Revista científica electrónica de Educación y Comunicación en la Sociedad del Conocimiento Etic@net, 1(14), (2014)

Guitert, M. y M. Pérez-Mateo; La colaboración en la red: Hacia una definición de aprendizaje colaborativo en entornos virtuales, http://www.redalyc.org/pdf/2010/201025739004.pdf, E-ISSN: 1138-9737, Teoría de la Educación. Educación y Cultura en la Sociedad de la Información (TESI), 14(1), 10-31 (2013)

Hernández, S.J., O.M. Quejada y G.M Díaz; Guía metodológica para el desarrollo de ambientes educativos virtuales accesibles: Una visión desde un enfoque sistémico, https://dialnet.unirioja.es/servlet/articulo?codigo=5580048, E-ISSN: 2013-9144, Digital Education Review, 29, 166-180 (2016)

Liu, K., R.J. Clarke, P.B. Andersen y R.K. Stamper; Coordination and Communication Using Signs: Studies in Organisational Semiotics, 2(1), Springer Science \& Business Media, New York, USA (2012)

Mascheroni, M. y C. Greiner; Calidad de software e ingeniería de usabilidad, XIV Workshop de Investigadores en Ciencias de la Computación, 656-659, Posadas, Argentina 26 y 27 de abril (2012)

Melo-solarte, D.S., y A.J. Uribe; KaVa Tutor: autorregulación y estimulación de procesos académicos en plataformas educativas, DOI: http://doi.org/10.5753/CBIE.SBIE.2013.818, SBIE - Simpósio Brasileiro de Informática na Educação, 24(1), 818-827 (2013)

Moll, S.; Gamificación: 7 claves para entender qué es y cómo funciona, http://justificaturespuesta.com/gamificacion-7claves-para-entender-que-es-y-como-funciona/, acceso: agosto 2017. (2014)

Mueller, D. y S. Strohmeier; Design characteristics of virtual learning environments: state of research, DOI: http://doi.org/10.1016/j.compedu.2011.06.017, Computers \& Education, 57(4), 2505-2516 (2011) 
Picard, R.W., S. Papert y otros 8 autores; Affective learning - a manifesto, DOI:

http://doi.org/10.1023/B:BTTJ.0000047603.37042.33, BT Technology Journal, 22(4), 253-268 (2004)

Posada, F.; Gamificación educativa, http://canaltic.com/blog/?p=1733, acceso: agosto 2017, CanalTIC (2013)

Ruiz, R.H.; Ambientes afectivos y efectivos de aprendizaje,

(https://www.uv.mx/personal/rubhernandez/files/2012/02/Amb_afectivos_efectivos.pdf , acceso: agosto 2017, Dimensiones Del Aprendizaje (2006)

Salinas, J.; Diseño y moderación de entornos virtuales de aprendizaje (EVA), DOI: http://doi.org/10.7238/rusc.v9i1.1301, Universities and Knowledge Society Journal (RUSC), 9(1), 194 (2012)

Sánchez, F.J.; Gamificación, DOI: http://doi.org/10.14201/eks20151621315, Education in the Knowledge Society (EKS), 16(2), 13-15 (2015)

Valencia, L.H.; El Aprendizaje Afectivo, (Módulo educativo construcción de ambientes virtuales innovadores), Manizales (2014)

Valverde, J. y M. Garrido; La funcion tutorial en entornos virtuales de aprendizaje: comunicación y comunidad, https://dialnet.unirioja.es/servlet/articulo?codigo=1303758, E-ISSN: 1695-288X, Revista Latinoamericana de Tecnología Educativa (RELATEC), 4(1), 153-167 (2012) 